\title{
AACR 2018 : l'océan source de nouvelles molécules anticancer
}

\author{
AACR 2018: the Ocean Source of New Anticancer Molecules
}

\author{
D. Grazziotin-Soares · J.-P. Lotz
}

(C) Lavoisier SAS 2018

Les composés d'origine marine constituent une source importante de substances naturelles. Cette source extrêmement diversifiée reste néanmoins peu exploitée, la plupart des composés naturels étant issus de plantes ou de micro-organismes terrestres. Dans le domaine des médicaments anticancéreux, quelques composés extraits d'organismes marins se sont pourtant déjà révélés très prometteurs. C'est le cas par exemple de l'ecteinascidine-743 (trabectédine, Yondelis ${ }^{\circledR}$ ), approuvée depuis une dizaine d'années pour traiter en deuxième ligne des patients atteints de sarcome des tissus mous après échec des anthracyclines et du cyclophosphamide. Compte tenu de leurs caractéristiques chimiques uniques, les composés d'origine marine ont également servi au design de nouveaux conjugués anticorps-médicaments (ADC pour antibody drug conjugate). Récemment, le PM050489, un composé marin capable de lier la tubuline sur un site distinct de celui de la vinblastine, a ainsi été conjugué au trastuzumab, et l'ADC résultant, appelé MI130004, étudié in vitro et in vivo sur des cellules exprimant différents niveaux de HER-2 [1].

Historiquement, et en raison du rôle essentiel des microtubules dans de nombreuses fonctions cellulaires, les microtubules ont souvent constitué une cible attractive pour le développement de nouveaux médicaments anticancéreux. En effet, leur instabilité dynamique détermine notamment la fixation correcte des chromosomes au fuseau mitotique et agit sur leur alignement lors de la métaphase et leur sépa-

\section{Grazziotin-Soares $(\bowtie)$}

Service d'oncologie médicale,

Alliance pour la recherche en cancérologie (Aprec),

hôpital Tenon, hôpitaux universitaires de l'Est-Parisien (AP-HP),

4, rue de la Chine, F-75970 Paris, France

e-mail : daniele.grazz@cancer-aprec.com

\section{J.-P. Lotz $(\square)$}

Service d'oncologie médicale et de thérapie cellulaire,

Alliance pour la recherche en cancérologie (Aprec),

institut universitaire de cancérologie,

université Pierre-et-Marie-Curie, hôpital Tenon,

hôpitaux universitaires de l'Est-Parisien (AP-HP),

4, rue de la Chine, F-75970 Paris, France

e-mail : jean-pierre.lotz@aphp.fr ration lors de l'anaphase. En clinique, on compte parmi les agents liant la tubuline, les alcaloïdes vinca et les taxanes. Ces agents induisent un arrêt mitotique prolongé au niveau de la transition métaphase/anaphase et induisent à terme la mort cellulaire par apoptose. Récemment, le cabazitaxel (un taxane), l'ixabepilone (une épothilone) et l'éribuline (un dérivé de l'homohalicondrine) ont été introduits en clinique pour traiter les cancers de la prostate et du sein. Le brentuximab vedotin, un ADC liant l'anticorps monoclonal chimérique brentuximab à la monométhyle auristatine $\mathrm{E}$ (d'origine marine), a également été développé et est aujourd'hui utilisé pour traiter le lymphome anaplasique à grandes cellules et le lymphome hodgkinien. Enfin, le trastuzumab emtansine, un ADC incorporant le trastuzumab et l'agent inhibiteur des microtubules DM1, a récemment été approuvé pour le traitement des patientes atteintes d'un cancer du sein avancé HER2-positif. Ensemble, ces données confirment l'intérêt continu que représentent les microtubules comme cible en chimiothérapie anticancéreuse.

Cette année à l'AACR, un nouveau composé cytotoxique d'origine marine et capable de perturber la dynamique du réseau microtubulaire a été présenté, la plocabuline [2]. Cette molécule, initialement isolée à partir d'une éponge marine appelée Lithoplocamia lithistoides, est désormais produite par synthèse chimique totale.

La plocabuline (PM060184) a récemment été évaluée dans deux modèles de xénogreffes dérivées de patients (PDX) atteints de tumeurs stromales-gastro-intestinales (GIST), caractérisées par une sensibilité variable à l'imatinib. Des souris ont ainsi été transplantées par des xénogreffes humaines IMA-sensibles ou IMA-résistantes. Les animaux xénogreffés ont ensuite été répartis aléatoirement dans trois groupes expérimentaux : témoin, imatinib et plocabuline. Le traitement a duré 22 jours, et l'activité antitumorale a été évaluée par la mesure du volume tumoral, l'histopathologie, la voie de signalisation KIT identifiée par Western blot et la réponse histologique $(\mathrm{RH})$. Cette dernière reposait sur les résultats macroscopiques et microscopiques de nécrose et de fibrose et a été notée pour chaque nodule tumoral : quatre réponses élevées, trois modérées et une minime ont été observées. 
Le traitement par plocabuline a entraîné une réduction du volume tumoral de $41 \%$ dans le modèle GIST-sensible et de $30 \%$ du volume de base dans le modèle GIST-résistante. Une bonne RH (grades 3,4) a été observée dans $70 \%$ (GIST-sensible) et $50 \%$ (GIST-résistante) des cas de tumeurs. La RH obtenue après traitement par la plocabuline se caractérisait principalement par une nécrose, tandis que l'imatinib produisait principalement une dégénérescence myxoïde dans le modèle sensible. Dans le modèle GISTrésistant, la plocabuline a montré une meilleure activité que l'imatinib en termes de réduction du volume tumoral (41 contre $146 \%, p<0,01$ ) et RH (grades 3, 4 chez 50 vs $0 \%$. La voie de signalisation KIT n'a, de son côté, pas été affectée par le traitement par la plocabuline. Le médicament expérimental a par ailleurs été bien toléré à la dose administrée. La plocabuline est le premier agent antitubuline à présenter une activité antitumorale sur des modèles de GISTPDX, tant sensible que résistant à l'imatinib. Compte tenu des différents modes d'action de la plocabuline et des TKIs (tyrosine kinase inhibitors), classe dont fait partie l'imatinib, les auteurs suggèrent de combiner plocabuline et imatinib pour surmonter l'apparition de résistance aux TKIs.
Travailler avec des composés marins est une approche relativement nouvelle dans la recherche en cancérologie. L'écosystème marin est pourtant une source riche de nouvelles entités chimiques qui peuvent également aider à identifier de nouvelles cibles cellulaires pour de futures interventions thérapeutiques. Ces nouvelles molécules fonctionnent par ailleurs au travers de mécanismes d'action uniques qui les distinguent de toutes les chimiothérapies conventionnelles, ce qui fait du monde marin une source extrêmement pertinente pour rechercher de nouveaux médicaments.

\section{Références}

1. Avilés P, Domínguez JM, Guillén MJ, et al (2018) MI130004, a novel antibody-drug conjugate combining trastuzumab with a molecule of marine origin, shows outstanding in vivo activity against HER2-expressing tumors. Mol Cancer Ther 17:786-94

2. Wozniak A, Wang Y, Wellens J, et al (2018) Plocabulin, a tubulin inhibitor, presents antitumor activity in patient-derived xenograft (PDX) models of gastrointestinal stromal tumor (GIST) [Abstract 3840, Session PO.ET06.11 - Novel and Canonical Targets], AACR 2018 